TRANSACTIONS OF THE

AMERICAN MATHEMATICAL SOCIETY

Volume 354, Number 9 , Pages 3757-3769

S 0002-9947(02)03008-8

Article electronically published on April 23, 2002

\title{
DEGENERATE FIBRES \\ IN THE STONE-ČECH COMPACTIFICATION OF THE UNIVERSAL BUNDLE OF A FINITE GROUP
}

\author{
DAVID FELDMAN AND ALEXANDER WILCE
}

\begin{abstract}
Applied to a continuous surjection $\pi: E \rightarrow B$ of completely regular Hausdorff spaces $E$ and $B$, the Stone-Čech compactification functor $\beta$ yields a surjection $\beta \pi: \beta E \rightarrow \beta B$. For an $n$-fold covering map $\pi$, we show that the fibres of $\beta \pi$, while never containing more than $n$ points, may degenerate to sets of cardinality properly dividing $n$. In the special case of the universal bundle $\pi: E G \rightarrow B G$ of a $p$-group $G$, we show more precisely that every possible type of $G$-orbit occurs among the fibres of $\beta \pi$. To prove this, we use a weak form of the so-called generalized Sullivan conjecture.
\end{abstract}

\section{INTRODUCTION}

This paper concerns the interaction of two fundamental, but at first glance unrelated, universal topological constructions: the classifying space, $B G$, of a group $G$ and the Stone-Čech compactification, $\beta X$, of a completely regular topological space $X$. The former belongs to algebraic topology, the latter to general topology. Why consider them together? We were initially motivated by the simple question of whether an $n$-to- 1 covering map always admits an $n$-to- 1 compactification. As it turns out, any compactified universal $G$-bundle $\beta E G \rightarrow \beta B G$ for a non-trivial finite group $G$ gives a counterexample.

Applied to a continuous surjection $\pi: E \rightarrow B$ of completely regular Hausdorff spaces, the Stone-Cech compactification functor $\beta$ yields a continuous surjection $\beta \pi: \beta E \rightarrow \beta B$. In the simple case where $E=B \times F, F$ a finite set, and $\pi$ is projection on the first factor, elementary considerations show that $\beta E=\beta B \times F$ and $\beta \pi$ is again projection on the first factor. However, as we shall show, nontrivial phenomena can arise in the slightly more general situation of an $n$-fold covering map $\pi$ (a local homeomorphism $\pi: E \rightarrow B$ with $\left|\pi^{-1}(b)\right|=n$ for any $b \in B$ ). Indeed, the fibres of $\beta \pi$, while never exceeding $n$ points, can degenerate to sets whose cardinality properly divides $n$. (Contrast this with the more usual, explosive sort of Stone-Cech "pathology".)

This phenomenon depends on a homotopy invariant, the sectional category, of the map $\pi$. Indeed, we show that for any $G$-bundle $\pi: E \rightarrow B, G$ a finite group, $\beta \pi$ has degenerate fibres exactly when $\pi$ has infinite sectional category.

In the special case of a universal $G$-bundle $\pi: E G \rightarrow B G$ for a finite $p$-group $G$, we obtain a more precise result: every possible type of $G$-orbit occurs among the fibres of $\beta \pi$. To prove this, we use a weak form of the so-called "generalized Sullivan

Received by the editors January 1, 2002.

2000 Mathematics Subject Classification. Primary 54D35, 55R35.

(C)2002 American Mathematical Society 
conjecture" [5], 1], now a theorem of H. Miller. A G-orbit, up to isomorphism, has the form $G / H$ for some subgroup $H$ of $G$. Thus, at least for $p$-groups, the lattice of subgroups of the structure group manifests itself in the geometry of the compactified universal bundle - this in spite of the fact that action of the group does not belong to the data fed to the Stone-Cech functor. In effect, the maximal compactification process automatically detects the symmetry of the bundle.

This work lies at the junction of algebraic and general topology, two very nearly autonomous mathematical disciplines. Accordingly, we have included enough detail throughout to make the paper essentially self-contained. In particular, we provide the necessary background on the Stone-Čech functor and homotopy theory (specifically, sectional category and homotopy fixed points). Readers unfamiliar with universal $G$-bundles should bear in mind the simplest non-trivial example, $G=\mathbb{Z} / 2 \mathbb{Z}$. The double cover of the infinite real projective space $\mathbb{R} \mathbb{P}^{\infty}$ forms a universal $\mathbb{Z} / 2 \mathbb{Z}$-bundle. No other finite group has a universal bundle so easily pictured; this case also motivates some of our terminology.

\section{BACKGROUND ON $\beta$}

We record here some basic lore about Stone-Čech compactifications. For further information, see [8].

Let $C(X)$ denote the ring of real-valued continuous functions on a topological space $X$. The space $X$ is said to be completely regular if $C(X)$ separates points from closed sets, 1 and Tychonoff if it is both completely regular and Hausdorff. We shall call a space locally Tychonoff if every point has a Tychonoff neighborhood. A locally Tychonoff Hausdorff space need not be Tychonoff (an example is the halfdisc topology on the upper half-plane [7]); however, it is easy to see that a locally Tychonoff regular space is Tychonoff.

For a function $f \in C(X), \mathbf{Z}(f)$ denotes $f^{-1}(0)$, the zero-set of $f$, and $\mathbf{C z}(f)$ denotes $X \backslash \mathbf{Z}(f)$, the cozero-set of $f$. We write $\mathbf{Z}[X]$ for the family of all zerosets in $X$. Finite unions and intersections of zero-sets are again zero-sets, since $\mathbf{Z}(f) \cup \mathbf{Z}(g)=\mathbf{Z}(f g)$ and $\mathbf{Z}(f) \cap \mathbf{Z}(g)=\mathbf{Z}(|f|+|g|)$. (Notice also that $\{x \mid f(x) \geq$ $0\}=\mathbf{Z}(\min \{f, 0\})$.)

By a $z$-filter on $X$ we mean a non-empty, proper subfamily $\mathfrak{F}$ of $\mathbf{Z}[X]$ closed under finite intersection and enlargement (meaning that $B \in \mathfrak{F}$ whenever $B \in \mathbf{Z}[X]$, $A \in \mathfrak{F}$ and $A \subseteq B$ ). By a $z$-ultrafilter we mean a maximal $z$-filter. Customarily, one denotes ultrafilters by small letters $u, v$, etc. Note that for any finite family $\left\{u_{i}\right\}$ of distinct $z$-ultrafilters, one can find a pairwise disjoint family $\left\{A_{i}\right\}$ of zero-sets such that $A_{i} \in u_{i}$.

Denote by $\beta X$ the collection of all $z$-ultrafilters on $X$. For a zero-set $F$ of $X$, let $\hat{F}=\{u \in \beta X \mid F \in u\}$. Topologize $\beta X$ by taking as a basis of closed sets the collection $\{\hat{F} \mid F \in Z[X]\}$. Let $i: X \rightarrow \beta X$ denote the natural map taking $x \in X$ to the principal $z$-ultrafilter $i(x)=\{F \in \mathbf{Z}[X] \mid x \in F\}$.

When $X$ is Tychonoff, the map $i$ is a homeomorphism onto its image; accordingly, we identify $X$ with $\beta X$. In this case, $\beta X$ compactifies $X-$ i.e., $\beta X$ is a compact Hausdorff space and $X$ is dense in $\beta X$. In fact, $\beta X$ provides a universal compactification of $X$, in the sense that any continuous mapping $X \rightarrow Y$, where $Y$ is compact Hausdorff, extends uniquely to a continuous mapping $\beta X \rightarrow Y$.

\footnotetext{
${ }^{1}$ This means that, for a closed set $A \subset X$ and a point $x \in X \backslash A$, there exists $f \in C(X)$ such that $f(x)=0$ and $f(A)=1$.
} 
Given a continuous map $f: X \rightarrow Y$, we define $\beta f: \beta X \rightarrow \beta Y$ by setting

$$
(\beta f)(u)=\left\{F \in \mathbf{Z}(Y) \mid f^{-1}(F) \in u\right\} .
$$

Clearly $\beta(f \circ g)=\beta f \circ \beta g$. Thus, $\beta$ is a functor from completely regular spaces to compact Hausdorff spaces.

Lemma 2.1. If $\pi: E \rightarrow B$ is a surjection between Tychonoff spaces $E$ and $B$, the map $\beta \pi: \beta E \rightarrow \beta B$ is also surjective.

Proof. Consider a surjection of Tychonoff spaces, $\pi: E \rightarrow B$. From the compactness of $\beta E$, we have the image of $\beta \pi$ compact, hence closed. But the image of $\beta \pi$ contains $B$, a dense subset of $\beta B$; so the image must coincide with $\beta B$.

\section{Stone-Čech Compactification of $G$-Bundles}

For the remainder of this paper $G$ denotes a finite group of order $n>1$ with identity element $e$. By a $G$-bundle, we always mean a covering map $\pi: E \rightarrow B$ with $E$ a $G$-space, $B=E / G$ and $\pi$ the canonical surjection taking each point $x \in E$ to its orbit.

We shall apply the Stone-Čech functor $\beta$ to such a $G$-bundle. The action of $G$ extends naturally to an action on $\beta E$. As we shall see, $G$ continues to act transitively on the fibres of $\beta \pi: \beta E \rightarrow \beta B$. A fibre will therefore have fewer than $n$ points iff it contains a $z$-ultrafilter fixed by some non-trivial element of $G$. The main results of this section (Theorems 3.4 and 3.7) give conditions under which a non-trivial subgroup of $G$ fixes a $z$-ultrafilter in $\beta E$.

Henceforth we assume that both the spaces $E$ and $B$ are Tychonoff, so that applications of the Stone-Čech functor will yield compactifications. Actually, in the context of Hausdorff spaces, it suffices to have $B$ Tychonoff:

Lemma 3.1. Suppose that $B$ is Tychonoff, $E$ is Hausdorff and $\pi: E \rightarrow B$ is a local homeomorphism. Then E Tychonoff. 2

Proof. It suffices to show that $E$ is regular, since the local homeomorphism $\pi$ guarantees $E$ locally Tychonoff. We apply the characterization of regular spaces as spaces in which all points have neighborhood bases consisting of closed sets. Fix a point $x$ of $E$ and an open neighborhood $U$ of $x$ small enough to make $\left.\pi\right|_{U}$ a homeomorphism. A neighborhood basis of closed sets $\left\{N_{i}\right\}_{i \in I}$ for the point $\pi(x)$ in $\pi(U)$ yields a neighborhood basis $\left\{U \cap \pi^{-1}\left(N_{i}\right)\right\}_{i \in I}$ for $x$. The regularity of $E$ will follow if we show that each set $\widetilde{N}_{i}=U \cap \pi^{-1}\left(N_{i}\right)$ is closed, not just in $U$, but in $E$.

Observe that for a set $N$ closed in $U, N \subset \pi^{-1}(\pi(N))$, a set closed in $E$, and therefore $y \in \operatorname{cl}(N)$ implies $y \in \pi^{-1}(\pi(N))$, or equivalently, $\pi(y) \in \pi(N)$.

Applying this observation with $N=\widetilde{N}_{i}$ shows that $y \in \operatorname{cl}\left(\widetilde{N}_{i}\right)$ implies $\pi(y) \in N_{i}$. Thus there exists $y^{\prime} \in \widetilde{N}_{i}$ such that $\pi\left(y^{\prime}\right)=\pi(y)$. We can now show that $\widetilde{N}_{i}$ is closed in $E$ by showing $y^{\prime}=y$.

Assume not. Then there exist disjoint open sets $V$ and $V^{\prime}$ in the Hausdorff space $E$ with $y \in V$ and $y^{\prime} \in V^{\prime}$. Now $y \in \operatorname{cl}\left(\widetilde{N}_{i}\right)$ gives $y \in \operatorname{cl}\left(\widetilde{N}_{i} \backslash V^{\prime}\right) \cup \operatorname{cl}\left(V^{\prime}\right)$. But clearly $y \notin \operatorname{cl}\left(V^{\prime}\right)$, and applying the observation above with $N=\widetilde{N}_{i} \backslash V^{\prime}$ shows also that $y \notin \operatorname{cl}\left(\widetilde{N}_{i} \backslash V^{\prime}\right)$, a contradiction.

\footnotetext{
${ }^{2}$ The real line with the origin doubled shows the necessity of some hypothesis on $E$.
} 
Lemma 3.2. Fix a G-bundle $\pi: E \rightarrow B$. Then $G$ acts transitively on each fibre of the map $\beta \pi$. In particular, the fibres of $\beta \pi$ form $G$-orbits, and hence have cardinality at most $n$.

Proof. Given $z$-ultrafilters $u, v$ in $\beta E$ such that $v \neq g u$ for any $g \in G$, we shall show that $(\beta \pi)(v) \neq(\beta \pi)(u)$.

For each $g \in G$, choose $C_{g} \in v$ such that $C_{g} \notin g^{-1} u$. Then $C=\bigcap_{g \in G} C_{g} \in v$; so $\pi(C) \in(\beta \pi)(v)$. On the other hand, $g C \notin u$ for any $g \in G$ (since $g C \subseteq g C_{g}$ and $\left.g C_{g} \notin u\right)$. Since $G$ acts transitively on the fibres of $\pi, \pi^{-1}(\pi(C))=\bigcup_{g \in G} g C$. But as the $z$-ultrafilter $u$ does not contain any of the (finitely many) sets $g C, u$ does not contain this last set either. Thus, $\pi(C) \notin(\beta \pi)(u)$.

Definition 3.3. Let $H$ be a subgroup of $G$. We call a cozero-set $U \subset E H$ antipolar if $U \cap h U=\emptyset$ for some $h \in H$. We call a zero-set $F \subset E H$-equatorial if we have $F \cup h F=E$ for some $h \in H$, or equivalently, if $F$ forms the complement of an $H$-antipolar set.

Theorem 3.4. For a $z$-ultrafilter $u$ on $E$, the following conditions are equivalent:

1. Every element of $H$ fixes $u$.

2. u contains every $H$-equatorial set.

Proof. $1 \Rightarrow 2$ : Suppose $H$ fixes $u$. For an $H$-equatorial set $F$, choose $h \in H$ such that $F \cup h F=E$. The $z$-ultrafilter $u$ must then contain either $F$ or $h F$. But $H$ fixes $u$; so $h F \in u$ implies $F \in u$. Hence $F \in u$.

$2 \Rightarrow 1$ : Suppose that $h u \neq u$ for some $h \in H$. Then there exist disjoint zero-sets $C_{e} \in u$ and $C_{h} \in h u$. Then $C:=C_{e} \cap h^{-1} C_{h}$ forms a zero-set in $u$ disjoint from $h C$. Fix a non-negative function $f \in C(E)$ with zero-set $C$. Then we claim that $F=\{x: f(x) \geq f(h x)\} \cup\left\{x: f(x) \geq f\left(h^{-1} x\right)\right\}$ gives an $H$-equatorial zeroset not in $u$. Certainly $F \cup h F=E$, since if $x \in E \backslash F$, then $f(x)<f\left(h^{-1} x\right)$, whence $h^{-1} x \in F$, i.e., $x \in h F$. We shall show that $F \notin u$ by demonstrating that $F \cap C=\emptyset$. Observe that the non-negative function $f \circ h^{-1}$ has zero-set $h C$. Since $C \cap h C=\emptyset, f$ and $f \circ h^{-1}$ never vanish simultaneously; after composing with $h$, we can say the same for $f \circ h$ and $f$. But for any $x \in F \cap C$, we must have either $0=f(x) \geq f(h x) \geq 0$, whence $f(h x)=0$, or $0=f(x) \geq f\left(h^{-1} x\right) \geq 0$, whence $f\left(h^{-1} x\right)=0$. This is a contradiction in either case, and so $F \cap C=\emptyset$.

Corollary 3.5. $H$ fixes points of $\beta E$ if and only if the class of $H$-equatorial sets has the finite intersection property.

Proof. If the $H$-equatorial sets have the finite intersection property, then they generate a $z$-filter, which we may extend to a $z$-ultrafilter.

Definition 3.6. Call a cozero-set $U \subset E H$-sectional if $U \cap h U=\emptyset$ for all $h \in H \backslash\{e\}$. Call a zero-set $F \subset E H$-cosectional if $F \cup h F=E$ for all $h \in H \backslash\{e\}$, or equivalently, if $F$ forms the complement of an $H$-sectional set.

Remark. For $\pi_{H}: E \rightarrow E / H$ the natural quotient map, we may characterize $H$ sectional subsets of $E$ as the images of sections of $\pi_{H}$ over cozero subsets of $E / H$.

Theorem 3.7. For a $z$-ultrafilter $u$ on $E$, the following conditions are equivalent:

1. Some element $h \in H \backslash\{e\}$ fixes $u$.

2. $u$ contains all $H$-cosectional sets. 
Proof. $1 \Rightarrow 2$ : Suppose $h u=u$ for some $h \in H \backslash\{e\}$. For an $H$-cosectional set $F$, $F \cup h F=E$. The $z$-ultrafilter $u$ must then contain either $F$ or $h F$. Since $h u=u$, $h F \in u$ implies $F \in u$; so $F \in u$ either way.

$2 \Rightarrow 1$ : Suppose no $h \in H \backslash\{e\}$ fixes $u$. We shall produce an $H$-cosectional set $F \notin u$. Our assumption guarantees that the ultrafilters $h u, h$ running over $H$, are distinct. Hence, there exist pairwise disjoint zero-sets $G_{h} \in h u, h \in H$. Since $h^{-1} G_{h} \in u$, the zero-set $C=\bigcap_{h \in H} h^{-1} G_{h}$ belongs to $u$, and $C \cap h C=\emptyset$ for every $h \in H \backslash\{e\}$.

Now fix a non-negative function $f \in C(E)$ with zero-set $C$. Since $C \cap h^{-1} C=\emptyset$,

$$
f(x)=0 \Rightarrow x \in C \Rightarrow x \notin h^{-1} C \Rightarrow h x \notin C \Rightarrow f(h x)>0
$$

for all $h \in H \backslash\{e\}$. Define zero-sets

$$
F_{h}=\{x: f(x) \geq f(h x)\} \text { and } F=\bigcup_{h \in H \backslash\{e\}} F_{h} .
$$

Then $F \cap C=\emptyset$, since $x \in C \Rightarrow 0=f(x)<f(h x) \Rightarrow x \notin F_{h}$. So $F \notin u$. We claim that $F$ is $H$-cosectional, i.e., $F \cup h F=E$ for all $h \in H \backslash\{e\}$. For if $x \in E$, then either $f(x) \geq f\left(h^{-1} x\right)$, in which case $x \in F_{h^{-1}} \subseteq F$, or $f\left(h^{-1} x\right) \geq f(x)=f\left(h\left(h^{-1} x\right)\right)$, in which case $h^{-1} x \in F_{h} \subseteq F$, whence $x \in h F_{h} \subset h F$.

Corollary 3.8. $H$ fails to act freely on $\beta H$ if and only if the class of $H$-cosectional sets has the finite intersection property.

\section{Fibre Degeneration and Sectional Category}

One calls a $G$-bundle $\pi: E \rightarrow B$ principal if $G$ acts freely on $E$. One calls a locally trivial bundle $\pi: E \rightarrow B$ numerable when one can trivialize it by an open cover of $B$ that supports a locally finite partition of unity; see [3]. The class of numerable bundles includes all locally trivial bundles over a paracompact base.

One calls a numerable principal $G$-bundle $\pi: E G \rightarrow B G$ universal if for any numerable principal $G$-bundle $q: E \rightarrow B$ there exists a map $\phi: B \rightarrow B G$, unique up to homotopy, such that $q=\phi^{*} p$. Universal $G$-bundles have contractible total spaces $E G$ - indeed, this characterizes universal $G$-bundles among $G$-bundles.

In this section, we prove the following.

Theorem 4.1. For any universal principal $G$-bundle $\pi_{G}: E G \rightarrow B G$ with $B G$ paracompact and for any subgroup $H \neq\{e\}$ of $G, \beta E G$ contains points fixed by a non-trivial subgroup of $H$.

The proof exploits the notion of the sectional category of a bundle $\pi: E \rightarrow B$. This is the minimum cardinality of an open cover of $B$ with $\pi$ admiting a section over each set in the cover; see the standard reference [6], which discusses the identical concept under the name genus 3 The term sectional category originated with James' survey of the Lusternik-Schnirelmann category [4].

\footnotetext{
${ }^{3}$ Many arguments about fibre bundles hinge at some point on the availability of a partition of unity (or something similar) and therefore require suitable hypotheses. Authors deal with this technicality in different ways. The approach of Švarc in [ 6] employs a nonstandard definition of open covering more restrictive than the usual one. Sectional category in our sense thus generally only bounds sectional category in his sense from below. The notion of a numerable bundle serves a similar purpose. Commonly one just assumes all base spaces to be paracompact.
} 
Lemma 4.2. Given a $G$-bundle $\pi: E \rightarrow B$, write $\pi_{H}: E \rightarrow E / H$ for the canonical quotient map. Then the $H$-cosectional sets generate a non-trivial filter iff $\pi_{H}$ has infinite sectional category.

Proof. Corollary [3.8 says that $\beta E$ has points fixed by a non-trivial element of $H$ iff the collection of $H$-cosectional sets has the finite intersection property, or equivalently, iff $E$ admits no covering by finitely many $H$-sectional sets. But as remarked in the previous section, a set $U \subseteq E$ is $H$-sectional iff the canonical quotient map $\pi_{H}: E \rightarrow E / H$, itself an $H$-bundle, has a section over $\pi_{H}(U)=$ $U / H$.

We now collect some facts concerning sectional category that we shall use in the proof of Theorem 4.1 and the sequel.

The $G$-category of a $G$-bundle $\pi: E \rightarrow B$ is the minimum cardinality of an open cover of $B$ by sets over which $\pi$ is trivial as a $G$-bundle. For principal $G$ bundles, sectional category and $G$-category coincide (since precisely trivial principal $G$-bundles admit sections).

The Lusternik-Schnirelmann category of a space $X$ (L-S category for short) equals the minimum cardinality of an open cover $\left\{U_{i}\right\}$ with each $U_{i}$ contractible in $X$. For the homotopy invariance of L-S category, see Prop. (1.1) of [4].

Lemma 4.3. The G-category of any universal principal $G$-bundle $\pi: E \rightarrow B$ coincides with the $L$-S category of $B$.

Proof. The bundle $\pi$ admits a section over any contractible open set $U$ in $B$; so the L-S category of $B$ provides an upper bound for the $G$-category of $\pi$. For the opposite bound, note that the existence of a section of $\pi$ over $U$ implies that $U$ is contractible in $B$, since the uniqueness aspect of the universal property of the bundle makes the inclusion map from $U$ to $B$ homotopic to a constant map. (The restriction of the numerable bundle $\pi$ to $U$ certainly yields a numerable bundle, as would any pullback of any numerable bundle.)

Thus, all universal principal $G$-bundles over $B$ have the same sectional category. Given a bundle $\pi: E \rightarrow B$, we now describe its $n$-fold fibre join $\pi^{(n)}: E^{(n)} \rightarrow B$. A point $x$ in $E^{(n)}$ with $\pi^{(n)}(x)=b$ is represented by an $n$-tuple $\left(t_{1} e_{1}, \ldots, t_{n} e_{n}\right)$, where the non-negative real numbers $t_{1}, \ldots, t_{n}$ (called weights) satisfy $t_{1}+\cdots+t_{n}=$ 1 and the points $e_{i} \in E$ satisfy $\pi\left(e_{1}\right)=\ldots=\pi\left(e_{n}\right)=b$; and where it is understood that $t_{i} e_{i}$ is independent of $e_{i}$ when $t_{i}=0$ ([3] gives further details).

Fact 4.4 (Prop. (8.1) in [4]; see also [6]). Suppose $B$ is paracompact. Then $\pi$ : $E \rightarrow B$ has sectional category less than or equal to $n$ if and only if the $n$-fold fibre join $E^{(n)}$ admits a section.

For the reader's convenience, we recall the short proof. Given an open cover $U_{1}, \ldots, U_{n}$ of $B$ and a section $\sigma_{i}$ of $\pi$ over each set $U_{i}$, any partition of unity $\left\{t_{i}\right\}$ subordinate to $\left\{U_{i}\right\}$ yields a section of $\pi^{(n)}$, namely $b \mapsto\left(t_{1}(b) \sigma_{1}(b), \ldots, t_{n}(b) \sigma_{n}(b)\right)$ for $b \in B$. Conversely, given a section $\sigma$ of $\pi^{(n)}$, say $\sigma(b)=\left(t_{1}(b) \sigma_{1}(b), \ldots\right.$, $\left.t_{n}(b) \sigma_{n}(b)\right)$ for $b \in B$, we have a cover of $B$ by the open sets $U_{i}=\left\{b \mid t_{i}(b) \neq 0\right\}$, and, over each of these, a well-defined section of $\pi$ determined by $\sigma_{i}$. 
Fact 4.5 (Prop. 50 in [6]). If a discrete group $G$ contains elements of finite order, then the bundle $\pi: E G \rightarrow B G$ has infinite $G$-category 4

Proof of Theorem 4.1. The natural map $\pi_{H}: E G \rightarrow E G / H$ forms a universal principal $H$-bundle, since $H$ acts freely on the contractible space $E G$. (Recall here that the contractibility of their total spaces characterizes universal bundles.) By Lemma 4.3 and Fact 4.5. the finiteness of $H$ implies that $\pi_{H}: E G \rightarrow E G / H$ has infinite sectional category. By Lemma 4.2, the $H$-cosectional sets generate a non-trivial filter. By Corollary [3.8, $\beta E G$ contains points fixed by some non-trivial subgroup of $H$.

In particular, for $H$ prime cyclic, $H$ itself must fix some points of $\beta E G$. Thus for each such $H, \beta \pi$ has degenerate fibres containing no more than $[G: H]$ points.

\section{H-Fixed Points and an Auxiliary Bundle}

So far we have seen that the infinite sectional category of universal $H$-bundles $\pi$ : $E H \rightarrow B H$ entails that the $H$-cosectional subsets of $E G$ have the finite intersection property, and this in turn guarantees the existence of points in $\beta E G$ fixed by some subgroup of $H$, by Corollary 3.8 .

We could prove the existence of points in $\beta E G$ fixed by $H$ itself, using Corollary 3.5, if we knew that the family of $H$-equatorial sets in $E G$ had the finite intersection property, or equivalently, that $E G$ had no cover by finitely many $H$ antipolar sets. In this section, we formulate an equivalent condition in terms of a certain auxiliary bundle's having infinite sectional category.

Write $\Delta F$ for the simplex generated by a finite set $F 5$ If $F$ carries an action by $H$, so does $\Delta F$, in which case we may speak of $H$-antipolar subsets of $\Delta F$. We denote by $\lambda F$ the subcomplex of $\Delta F$ consisting of the union of all $H$-antipolar faces. Given an $H$-bundle $\pi: E \rightarrow B$, we may perform this construction on each fibre to obtain an $H$-bundle, which we denote by $\lambda \pi: \lambda E \rightarrow B[$ This is the auxiliary bundle alluded to above. In order to obtain the advertised result, we shall need to assume $B$ normal. We shall also need the following two technical lemmas.

Lemma 5.1. For the product $B \times F$ of a normal space $B$ and a discrete finite set $F$, write $\pi_{B}$ and $\pi_{F}$ for the projections from $B \times F$ onto $B$ and $F$. Given an open set $V \subseteq B \times F$ and a point $x \in B$, write $Q(x)$ for the set $\pi_{F}\left(\pi_{B}{ }^{-1}(x) \cap V\right)$. Then there exists a map $t: \pi_{B}(V) \rightarrow \Delta F$ such that $t(x) \in \Delta Q(x)$ for all $x \in \pi_{B}(V)$.

Proof. Set $U_{j}=\{x \in \pi(V):|Q(x)| \leq j\}$. For $x \in U_{1}$, set $t(x)$ equal to the unique element of $Q(x)$. We proceed by induction on $j$. Suppose $t$ is already defined on $U_{j}$ in such a way that for every $A \subset F$ with $|A| \leq j, t(x) \in \Delta A$ for all $x \in \operatorname{cl}\left(Q^{-1}(A)\right)$. We must extend $t$ continuously to $U_{j+1}$ so that for every $A \subset F$ with $|A|=j+1$ we have $t(x) \in \Delta A$ for all $x \in \operatorname{cl}\left(Q^{-1}(A)\right)$.

\footnotetext{
4 The proof of Prop. 50 in [6] contains a misprint: The reference to Theorem 10 should instead point to Theorem 17 .

${ }^{5}$ Thus, $\Delta F$ consists of functions $w \in[0,1]^{F}$ with $\sum_{x \in F} w(x)=1$. We identify elements of $F$ with the vertices of $\Delta F$, i.e., $x \in F$ is identified with the function that is 1 at $x$ and identically zero on $F \backslash\{x\}$. For any $w \in \Delta F$, we speak of $w(x)$ as the barycentric coordinates of $w$.

${ }^{6}$ The simplest way to do this is to begin with the $H$-bundle $p^{\prime}: E^{\prime} \rightarrow B$ whose fibre above $b \in B$ is $[0,1]^{p^{-1}(b)}$, topologized so that its continuous sections correspond to continuous mappings $f: E \rightarrow[0,1]$; the bundles $\Delta p: \Delta E \rightarrow B$ and $\lambda p: \lambda E \rightarrow B$ can be obtained as sub-bundles of this.
} 
Continuity of a function on the closed set $U_{j}$ and on each of the finitely many closed sets $\operatorname{cl}\left(Q^{-1}(A)\right) \cap U_{j+1}(A \subset F$ with $|A|=j+1)$ guarantees continuity on all of $U_{j+1}$. Thus we need only extend $t$ continuously from $\operatorname{cl}\left(Q^{-1}(A)\right) \cap U_{j}$ to $\operatorname{cl}\left(Q^{-1}(A)\right)$ with values in $\Delta A$ for each $A \subset F$ with $|A|=j+1$. The coordinate functions of $t$ associated with $v_{i} \in F \backslash A$ equal zero identically on $\operatorname{cl}\left(Q^{-1}(A)\right) \cap U_{j}$; so these we simply extend by zero. For the remaining coordinate functions, those associated to vertices in $A$, we apply the Tietze extension theorem. This yields a function $s: \operatorname{cl}\left(Q^{-1}(A)\right) \rightarrow[0,1]^{A}$ agreeing with $t$ on $\operatorname{cl}\left(Q^{-1}(A)\right) \cap U_{j}$. Finally, to extend $t$ to $\operatorname{cl}\left(Q^{-1}(A)\right)$, we compose $s$ with a retraction from $[0,1]^{A}$ onto $\Delta A$.

Lemma 5.2. Given $\pi: E \rightarrow B$, an $H$-bundle over a normal space $B$ with $H$ acting transitively on fibres, and $V$ an $H$-antipolar subset of $E$, there exists a partial section $t: \pi(V) \rightarrow \lambda E$ of the bundle $\lambda \pi: \lambda E \rightarrow B$ such that $t(x) \in \Delta\left(\pi^{-1}(x) \cap V\right)$ for all $x \in \pi(V)$.

Proof. The special case where $E$ has the form $B \times F$ for a transitive $H$-set $F$ follows immediately from Lemma 5.1. We now reduce the general case to this special case.

Denote by $E^{\prime}$ another copy of $E$. Consider the pullback

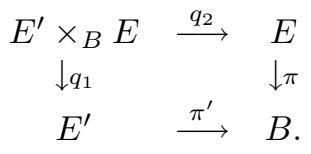

We regard the map $q_{1}: E^{\prime} \times_{B} E \rightarrow E^{\prime}$ as an $H$-bundle by letting $H$ act in the usual way on $E$, but trivially on $E^{\prime}$. Taking the diagonal map as a section trivializes the bundle $q_{1}: E^{\prime} \times_{B} E \rightarrow E^{\prime}$. Observe that $q_{2}^{-1}(V)$ forms an $H$ antipolar subset of $E^{\prime} \times_{B} E$. From Lemma 5.1] $\lambda q_{1}: \lambda\left(E^{\prime} \times_{B} E\right) \rightarrow E^{\prime}$ has a partial section $s: \pi^{\prime-1}(\pi(V)) \rightarrow \lambda\left(E^{\prime} \times_{B} E\right)$ such that $s(x) \in \Delta\left(q_{1}^{-1}(x) \cap q_{2}^{-1}(V)\right)$ for all $x \in \pi^{\prime-1}(\pi(V))$.

If we now let $H$ act in the usual way on $E^{\prime}$, but trivially on $E$, we may view the map $q_{1}: E^{\prime} \times_{B} E \rightarrow E^{\prime}$ as $H$-equivariant. There exists a canonical isomorphism between the quotient of $q_{1}$ by this action and the bundle $\pi: E \rightarrow B$. Thus, giving a partial section $t: \pi(V) \rightarrow \lambda E$ amounts to giving an $H$-equivariant partial section from $\pi^{\prime-1}(\pi(V))$ to $\lambda\left(E^{\prime} \times_{B} E\right)$.

While $H$-equivariance may fail for the partial section

$$
s: \pi^{\prime-1}(\pi(V)) \rightarrow \lambda\left(E^{\prime} \times_{B} E\right),
$$

we do get an $H$-equivariant map when we pass to the fibrewise barycenter $\bar{s}$ : $\pi^{\prime-1}(\pi(V)) \rightarrow \lambda\left(E^{\prime} \times_{B} E\right)$ of all the translates $h s h^{-1}$ of $s, h$ running over $H$. Clearly $\bar{s}(x) \in \Delta\left(q_{1}^{-1}(x) \cap q_{2}^{-1}(V)\right)$. The quotient of $\bar{s}$ by $H$ gives us a mapping $t: \pi(V) \rightarrow \lambda E$ with $t(x) \in \Delta\left(\pi^{-1}(x) \cap V\right)$ for all $x \in \pi(V)$, as desired.

Theorem 5.3. Given $\pi: E \rightarrow B$, an $H$-bundle over a normal space $B$ with $H$ acting transitively on the fibres, the following conditions are equivalent:

1. $\lambda \pi: \lambda E \rightarrow B$ has infinite sectional category.

2. E admits no cover by finitely many $H$-antipolar sets.

Proof. $1 \Rightarrow 2$ : Suppose that $E$ has a cover by finitely many $H$-antipolar sets $V_{i}$, in which case the open sets $U_{i}=\pi\left(V_{i}\right) \sqrt{7}$ cover $B$. Then Lemma 5.2 allows us to find a partial section $t_{i}$ of $\lambda \pi: \lambda E \rightarrow B$ over each $U_{i}$.

\footnotetext{
${ }^{7}$ Recall here that $\pi$ is a covering map, hence, open.
} 
$2 \Rightarrow 1$ : Suppose $\lambda \pi: \lambda E \rightarrow B$ has finite sectional category. Then $B$ has a finite open cover $\left\{U_{1}, \ldots, U_{n}\right\}$ such that $\lambda \pi: \lambda E \rightarrow B$ admits a partial section $s_{i}$ over each $U_{i}$. Each $s_{i}$ determines an $H$-antipolar open subset $V_{i}$ of $E$ with $V_{i} \cap \pi^{-1}(x)$ equal to the set of vertices of the smallest face containing $s_{i}(x)$. Then the $V_{i}$ and all their $H$-translates cover $E$.

Note that only the first implication requires assuming $B$ normal.

Remark 5.4. We might bypass Theorm 5.3 by working instead with a different auxiliary bundle, one having finite non-Hausdorff spaces as fibres. The fibre above $x$ consists of points $v_{i}$ to represent each of the $H$-antipolar subsets $V_{i} \subset \pi^{-1}(x)$; the closure of a particular $v_{i}$ consists of all the points representing subsets of the associated $V_{i}$.

\section{6. $p$-Groups and the Sullivan Conjecture}

Applying the results of the previous section to a universal bundle $\pi: E H \rightarrow B H$, we see that $\beta E H$ (and hence, $\beta E G$ ) has points fixed by $H$ iff $\lambda \pi$ has infinite sectional category. Unfortunately, computing the sectional category of the bundle $\lambda \pi: \lambda E H \rightarrow B H$ seems difficult due to the combinatorial complexity of the bundle itself; at present we do not know whether these bundles have infinite sectional category for every $H$. On the other hand, showing that a larger, but structurally simpler, bundle has infinite sectional category suffices for our purposes. This we can do when $H$ is a $p$-group.

First, for a finite $H$-space $F$, write $\Lambda F$ for the $H$-invariant subcomplex of $\Delta F$ consisting of all cells except the single cell of highest dimension corresponding to the set $F$ itself. Observe that the $H$-space $\Lambda F$ contains $\lambda F$ as a subcomplex, since we certainly never have $F$ itself $H$-antipolar in $F$. Now write $\Lambda \pi: \Lambda E H \rightarrow B H$ for the sub-bundle of $\Delta \pi: \Delta E H \rightarrow B H$ obtained by performing this construction on each fibre.

Henceforth assume $B H$ to be paracompact. We claim that $\Lambda \pi$ has infinite sectional category. In light of Fact 4.4 we just need to show that the $n$-fold fibre join $(\Lambda \pi)^{(n)}:(\Lambda E H)^{(n)} \rightarrow(B H)^{(n)}$ has no section for any $n$.

Fix a particular fibre $F$ of $\pi: E H \rightarrow B H$, enumerating the points of $F$ from 1 to $|F|$. We can then identify points of $\Lambda F$ with $n$-tuples $\left(s_{1}, \ldots, s_{|F|}\right)$, where $s_{i} \in[0,1]$ and $\sum_{i} s_{i}=1$, and where $s_{i}=0$ for at least one $i$. Accordingly, we can represent points of $\Lambda F^{(n)}$, the $n$-fold join of $\Lambda F$, by weighted $n$-tuples of the form

$$
\left(t_{1}\left(s_{1,1}, \ldots, s_{|F|, 1}\right), \ldots, t_{n}\left(s_{1, n}, \ldots, s_{|F|, n}\right)\right)
$$

satisfying

(i) $t_{j}, s_{i, j} \in[0,1]$ for $j=1, \ldots, n, i=1, \ldots,|F|$;

(ii) $\sum_{j=1}^{n} t_{j}=1$;

(iii) $\sum_{i=1}^{|F|} s_{i, j}=1$ for $j=1, \ldots, n$; and

(iv) for each $j$ there exists an $i$ such that $s_{i, j}=0$.

The representation is unique provided that we identify two such expressions precisely when they differ only at coordinates with weight $t_{j}=0$.

Now to each such weighted $n$-tuple associate the $n \times|F|$ matrix $\left|a_{i, j}\right|$, where $a_{i, j}=t_{j} s_{i, j}$. Observe that this matrix depends only on the point of $\Lambda F^{(n)}$ itself, not on the particular representation of the point by a weighted $n$-tuple, and that the matrix determines the point. Precisely those $n \times|F|$ matrices arise in this fashion that satisfy 
(i) each $a_{i, j} \in[0,1]$;

(ii) each row contains at least one entry equal to 0 ;

(iii) $\sum_{j=1}^{n} \sum_{i=1}^{|F|} a_{i, j}=1$.

Of course $H$ acts on the set of such matrices by permuting columns. This action can have no fixed points. Indeed, with the action of $H$ on $F$ transitive and a 0 in every row, a fixed matrix would have to vanish identically, contrary to (iii).

Pull $(\Lambda \pi)^{(n)}:(\Lambda E H)^{(n)} \rightarrow B H$ back along $\pi: E H \rightarrow B H$ to obtain the bundle

$$
\pi \times_{B H}(\Lambda \pi)^{(n)}: E H \times_{B H}(\Lambda E H)^{n} \rightarrow E H .
$$

The contractibility of $E H$ makes this bundle trivial, hence isomorphic (as an $H$ bundle) to the first factor projection

$$
\pi_{1}: E H \times(\Lambda H)^{n} \rightarrow E H .
$$

In other words, we have $(\Lambda \pi)^{(n)}:(\Lambda E H)^{(n)} \rightarrow B H$ isomorphic to

$$
\pi_{1} / H:\left(E H \times(\Lambda H)^{n}\right) / H \rightarrow E H / H .
$$

Thus a section of $(\Lambda \pi)^{(n)}:(\Lambda E H)^{(n)} \rightarrow B H$ amounts to an $H$-equivariant map $E H \rightarrow(\Lambda H)^{(n)}$.

On the other hand, an $H$-equivariant map from a point to $(\Lambda H)^{(n)}$ amounts to an $H$-fixed point. We just saw that the $H$-action on $(\Lambda H)^{(n)}$ has no fixed points. For a $p$-group $H$, Miller's version of the Sullivan conjecture [5] provides a homotopy equivalence between the homotopy fixed point space of $(\Lambda H)^{(n)}$, namely, $\operatorname{Hom}_{H}\left(E H,(\Lambda H)^{(n)}\right)$, and the fixed point space of $(\Lambda H)^{(n)}$. (See [1, Theorem A, for a result weaker than Miller's, easier to prove, and still sufficient for the present purpose.) Therefore $\operatorname{Hom}_{H}\left(E H,(\Lambda H)^{(n)}\right)=\emptyset$, or equivalently, there exists no $H$ equivariant map $E H \rightarrow(\Lambda H)^{(n)}$. We conclude that the bundle $\Lambda \pi: \Lambda E H \rightarrow B H$ has infinite sectional category, so we have proved

Theorem 6.1. For any universal $G$-bundle $E G \rightarrow B G$ with $B G$ normal and paracompact, and for any p-subgroup $H$ of $G, \beta E G$ has points fixed by $H$.

Searching for a weaker hypothesis on the subgroup $H$ leads, by previous considerations, to the following problem:

Problem 6.2. What groups $H$, not $p$-groups, have the homotopy fixed point space $\operatorname{Hom}_{H}\left(E H,(\lambda H)^{(n)}\right)$ empty for all $n$ ?

\section{Remarks on More General Groups}

Asking, as in the last section, for a larger bundle to have infinite sectional category amounts to asking for the finite intersection property for a larger family of zero-sets. Let us call a cozero-set $U \subset E H H$-incomplete if

$$
\bigcap_{h \in H} h U=\emptyset
$$

(meaning $U$ misses at least one element in each fibre over $B H$ ), and a zero-set $H$-coincomplete if it forms the complement of an $H$-incomplete set. Theorem 6.1 shows that for a $p$-group $H$ and a normal, paracompact classifying space $B H$, the $H$-incomplete sets generate a non-trivial filter in $B H$. Since the $H$-incomplete filter contains the $H$-equatorial filter, conceivably we might have the former trivial and the latter not. The following proposition gives a sufficient condition for these two filters to coincide. 
Proposition 7.1. For a finite group $H$, fix $k$ such that $k^{2}-k+1<|H|$. Suppose an $H$-incomplete set $U$ in a principal $H$-bundle misses no more than $k$ points from any fibre that it meets. Then $U$ admits a finite covering by $H$-antipolar sets.

Proof. Let

$$
\mathfrak{S}=\{S \subset H|| S|>| H \mid-k\} .
$$

For each $S \in \mathfrak{S}$, set $U_{S}=\bigcap_{h \in S} h U$.

Note that the cozero-sets $U_{S}$ cover $U$, since $p \in U$ implies $p \in U_{S}$ for $S=\{h \mid p \in$ $h U\}$.

Now we shall demonstrate the $H$-antipolarity of each $U_{S}, S \in \mathfrak{S}$; this means finding a $j \in H$ such that $U_{S} \cap j U_{S}=\emptyset$. Actually, any $j$ such that $S \cup j S=H$ suffices, since then $U_{S} \cap j U_{S}=U_{S} \cap U_{j S}=\bigcap_{h \in H} h U=\emptyset$, by the $H$-incompleteness of $U$.

To see why we have a $j \in H$ with $S \cup j S=H$, observe that $S \cup j S \neq H$ implies that $H \backslash S$ must contain an $x$ with $j^{-1} x \notin S$, so that $j=x\left(x^{-1} j\right) \in(H \backslash S)(H \backslash S)^{-1}$. But $|S|>|H|-k$ implies $\left|(H \backslash S)(H \backslash S)^{-1}\right| \leq k^{2}-k+1<|H|$.

On passing to complements, the proposition says that the equality of the $H$ coincomplete and the $H$-equatorial filters follows from the assumption that minimal $H$-coincomplete sets contain no more than $k$ points of those fibres they do not contain entirely.

Remark. One might consider properties intermediate between $H$-antipolarity and $H$-incompleteness in hopes of extending Theorem 6.1 to a larger class of groups. Some candidates:

a) $\bigcap_{h \in K} h U=\emptyset$ for some cyclic proper subgroup $K$;

b) $\bigcap_{h \in K} h U=\emptyset$ for some proper subgroup $K$;

c) $U$ meets no more than half the points in any orbit.

The last condition at least has combinatorial simplicity to recommend it.

\section{Loci of Degeneration}

The homotopy equivalence of all universal $G$-bundles notwithstanding, we can expect sensitivity of the Stone-Čech functor to topological differences between such bundles. We now obtain especially precise results by concentrating specifically on Milnor's well-known models of universal $G$-bundles. Given a group $G$, Milnor sets $E G$ equal to the infinite fibre join $G * G * G * \cdots$ (i.e., the direct limit of the fibre joins $\left.G^{(n)}\right)$; the quotient by the natural left action of $G$ then gives $B G$. For details, see [3].

Henceforth, we deal specifically with Milnor's model of the universal $G$-bundle. This offers two advantages. First, Milnor's $E G$ and $B G$ are metrizable. Secondly, for each subgroup $H$ of $G$, Milnor's $E G$ contains Milnor's $E H=H * H * H * \cdots$ as a closed subspace and, correspondingly, Milnor's $B G$ contains a closed subspace canonically homeomorphic to Milnor's $B H$, namely the quotient of this $E H$ by $H$. (Indeed, $B H$ actually has $|G / H|$ disjoint copies of $E H$ sitting over it: for each left coset $g H$ the infinite join $g H * g H * g H * \cdots$ gives one such.)

We measure the degeneration of a fibre of $\pi_{G}: \beta E G \rightarrow \beta B G$ by the conjugacy class of the stabilizer of any point in that fibre. Under the hypotheses of Theorem 8.1 (e.g., for $G$ a $p$-group), each conjugacy class of subgroups of $G$ has an associated non-empty locus in $B G$ where the fibres display just the corresponding 
sort of degeneration. We wish to emphasize again that the action of $G$ on $E G$ has no direct role in the data fed to the Stone-Čech functor; rather the compactification process automatically detects the symmetry of the bundle.

Theorem 8.1. Fix a finite group $G$. Suppose that for each subgroup $H$ of $G$ and each universal $H$-bundle $\pi_{H}: E H \rightarrow B H$ with $B H$ normal, $\beta \pi_{H}: \beta E H \rightarrow \beta B H$ has at least one fibre consisting of a single point. Then for each such $H, \beta E G$ has points with stabilizer exactly $H$.

We shall need a preliminary lemma. Given a family $\mathfrak{F}$ of zero-sets in a space $X$, let $\mathfrak{F}^{\prime}$ denote the collection of zero-sets having a non-empty intersection with every member of $\mathfrak{F}$. For $\mathfrak{F}$ a $z$-filter, $\mathfrak{F}^{\prime}$ just equals the union of the $z$-ultrafilters extending $\mathfrak{F}$. Note that if $\mathfrak{F} \subseteq \mathfrak{G}$, then $\mathfrak{G}^{\prime} \subseteq \mathfrak{F}^{\prime}$, and also that $\left(\bigcup_{i} \mathfrak{F}_{i}\right)^{\prime}=\bigcap_{i} \mathfrak{F}_{i}^{\prime}$ for any family $\mathfrak{F}_{i}$.

A perfectly normal space is one in which every closed set is a $z$-set. Every metrizable space - in particular, Milnor's $E G$ - is perfectly normal.

Lemma 8.2. For $\mathfrak{F}$ a $z$-filter on a perfectly normal space $X$, if a zero-set $C$ belongs to $\mathfrak{F}^{\prime \prime}$, then every closed neighborhood of $C$ belongs to $\mathfrak{F}$.

Proof. Fix $C \in \mathfrak{F}^{\prime \prime}$ and suppose $C \subseteq U \subseteq N$, with $U$ open and $N$ closed. Since $X \backslash N \subseteq X \backslash U, C \cap \operatorname{cl}(X \backslash N)=\emptyset$. Since $X$ is perfectly normal, $\operatorname{cl}(X \backslash N) \in \mathbf{Z}[X]$; so $\operatorname{cl}(X \backslash N) \notin \mathfrak{F}^{\prime}$. Thus $\mathfrak{F}$ must contain a set $E$ disjoint from $\operatorname{cl}(X \backslash N)$. Then from the fact that $E \subseteq N$ and the fact that $\mathfrak{F}$ is a filter we conclude that $N \in \mathfrak{F}$.

Remark 8.3. The converse of the lemma (of which we shall make no use) actually holds with no restriction on $X$ : for a $z$-filter $\mathfrak{F}$ on any space $X$, if every closed neighborhood of a zero-set $C$ belongs to $\mathfrak{F}$, then $C$ belongs to $\mathfrak{F}^{\prime \prime}$. Indeed, given a zero-set $C \notin \mathfrak{F}^{\prime \prime}$, there exists a zero-set $D \in \mathfrak{F}^{\prime}$ disjoint from $C$. Since the complete separation of disjoint zero-sets holds in any space, $C$ must have a closed neighborhood $N$ disjoint from $D$. But $N$ disjoint from $D \in \mathfrak{F}^{\prime}$ implies $N \notin \mathfrak{F}$, as desired.

Proof of Theorem 8.1. Suppose the theorem is false. This means that $G$ has a subgroup $H$ which does not occur as the stabilizer (in $G$ ) of any point of $\beta E G$. Write $\mathfrak{H}$ for the $H$-equatorial filter. Fix an enumeration $K_{1}, \ldots, K_{n}$ of the subgroups of $G$ that properly contain $H$, and write $\mathfrak{K}_{i}$ for the $K_{i}$-equatorial filter.

The free action of $H$ on $E G$ makes the bundle $E G \rightarrow E G / H$ a universal $H$ bundle with a normal base. By hypothesis, the bundle $\beta E G \rightarrow \beta(E G / H)=$ $(\beta E G) / H$ then has at least one fibre that consists of just a single point. Thus $\beta E G$ does possess $H$-stable points, which guarantees the non-triviality of $\mathfrak{H}$.

By the choice of $H$, the stabilizer of an $H$-stable point always contains $H$ properly; so the stabilizer of each $H$-stable point of $\beta E G$ lies among the $K_{i}$. Thus a $z$-ultrafilter extending $\mathfrak{H}$ must also extend some $\mathfrak{K}_{i}$. This means that $\mathfrak{H}^{\prime} \subseteq \bigcup_{i} \mathfrak{K}_{i}^{\prime}$, and thus $\bigcap_{i} \mathfrak{K}_{i}^{\prime \prime} \subseteq \mathfrak{H}^{\prime \prime}$.

Since $\mathfrak{K}_{i} \subset \mathfrak{K}_{i}^{\prime \prime}$, we can obtain a contradiction by showing that $\bigcap_{i} \mathfrak{K}_{i} \not \subset \mathfrak{H}^{\prime \prime}$.

As we noted, $E G$ contains a canonical copy of $E H$. This $E H$ and all its distinct $G$-translates $g_{k} E H$ lie over the canonical copy of $B H$ in $B G$, disjointly (assume here that $g_{1}=e$, the identity). By normality, the $g_{k} E H$ have disjoint open neighborhoods $U_{k}$. If necessary, replace $U=U_{1}$ by $\bigcap g_{k}^{-1} U_{k}$, making $U$ disjoint from $g U$ for any $g \notin H$. This makes $U K_{i}$-antipolar for each $i$. Then $E G \backslash U \in \mathfrak{K}_{i}$ for each 
$i$, whence $E G \backslash U \in \bigcap_{i} \mathfrak{K}_{i}$. To obtain the contradiction we seek, it now suffices to show that $E G \backslash U \notin \mathfrak{H}^{\prime \prime}$.

Since Lemma 8.2 says that a closed neighborhood of an element of $\mathfrak{H}^{\prime \prime}$ belongs to $\mathfrak{H}$, showing $E G \backslash U \notin \mathfrak{H}^{\prime \prime}$ reduces to finding a closed neighborhood of $E G \backslash U$ that does not belong to $\mathfrak{H}$. The normality of $E G$ yields disjoint open sets $W$ and $W^{\prime}$ with $E H \subseteq W$ and $E G \backslash U \subseteq W^{\prime}$. Certainly $E G \backslash W$ (which contains the closure of $W^{\prime}$ ) constitutes a closed neighborhood of $E G \backslash U$. We can finish now by showing that $E G \backslash W \notin \mathfrak{H}$, or equivalently, by showing that $W$ admits no covering by finitely many $H$-antipolar sets.

Observe that $E H \rightarrow E H / H=B H$ also constitutes a universal $H$-bundle. Reapplying the hypothesis, $\beta E H \rightarrow \beta B H$ has fibres consisting of a single point. This guarantees the non-triviality of the $H$-equatorial filter on $E H$. Thus we cannot cover $E H$ by finitely many $H$-antipolar sets. A fortiori, neither can we cover $W$ by finitely many $H$-antipolar sets.

\section{ACKNOWLEDGMENTS}

We owe a debt to Mike Hopkins for introducing us to the literature concerning sectional category and the Sullivan conjecture, thereby making a crucial contribution to this project. We also thank Raoul Bott, Wis Comfort, Art Copeland and Bob Heath for helpful discussions.

\section{REFERENCES}

[1] E. Dror Farjoun and A. Zabrodsky, Fixed points and homotopy fixed points, Comment. Math. Helvetici 63 (1988), 286-295. MR 90e:55025

[2] S. Eilenberg and T. Ganea, On the Lusternik-Schnirelmann category of abstract groups, Ann. Math. 65 (1957), pp. 517-518. MR 19:52d

[3] D. Husemoller, Fibre Bundles, 3rd edition, Springer-Verlag, 1993. MR 94k:55001

[4] I. M. James, On category, in the sense of Lusternik-Schnirelmann, Topology 17 (1978), pp. 331-348. MR 80i:55001

[5] H. Miller, The Sullivan conjecture on maps from classifying spaces. Annals of Math. 120 (1984), 39-87. Erratum: Annals of Math. 121 (1985), 605-609. MR 85i:55012 MR 87k:55020

[6] A. S. Švarc, The genus of a fibre space, Amer. Math. Soc. Transl. 55 (1966), pp. 49-140. MR 27:1963. MR 27:4233 (Russian originals)

[7] L. A. Steen and J. A. Seebach, Jr., Counterexamples in Topology, Holt, Rinehart and Winston, New York, 1970. MR 42:1040

[8] Russell C. Walker, The Stone-Čech Compactification, Springer-Verlag, 1974. MR 52:1595

Department of Mathematics, University of New Hampshire, Durham, New Hampshire 03824

E-mail address: David.Feldman@unh.edu

Department of Mathematics and Computer Science, Juniata College, Huntingdon, Pennsylvania 16652

E-mail address: wilce@juniata.edu

Current address: Department of Mathematical Sciences, Susquehanna University, Selinsgrove, PA 17870

E-mail address: wilce@susqu.edu 